\title{
La participación educativa como una herramienta de mejora
}

\author{
Educational participation as a tool for improvement
}

\section{Christian Loyola Bustos*}

\section{RESUMEN}

Actualmente, la participación corresponde a un proceso fundamental para generar procesos de mejora educativa efectiva, demostrada en una serie de marcos normativos y legales de la política educativa. Por eso asume importancia la Política Nacional de Convivencia Escolar y la Creación del Plan de Formación Ciudadana. El presente trabajo tiene como objetivo dar a conocer la importancia de la participación en los procesos de mejora educativa escolar. La metodología de trabajo se centró en la revisión de fuentes secundarias especializadas, poniendo énfasis en la importancia de la participación como herramienta de gestión efectiva. La evidencia permite concluir que fomentar una participación activa genera un desafío potente para los líderes educativos, en orden a movilizar creencias asociadas a la colaboración mutua entre los que están insertos en la organización escolar. Este desafío está en no plantear la participación educativa a partir de marcos normativos "impuestos", sea esta una ley o un decreto, sino que, por el contrario, las comunidades educativas busquen la promoción de acciones colaborativas participativas a partir de la autogestión, tomando sus contextos y criterios, favoreciendo la creación de altas expectativas, confianza y procesos de mejora.

\section{SUMMARY}

Currently, engagement is a fundamental process for generating effective instructional improvement methods, proved in a series of normative and legal frameworks of educational policy. That
Palabras clave:

liderazgo, participación educativa, mejora escolar, política nacional de convivencia escolar, formación ciudadana.

Key words: leadership, educational participation,

Profesor de Historia, licenciado en Historia. Magíster en Política Educativa y en Liderazgo y Gestión de las Organizaciones Escolares. Pontificia Universidad Católica de Valparaíso. Contacto: christian.loyola@pucv.cl ORCID: https://orcid.org/0000-0003-4792-9936 
is why the National Policy for School Coexistence and the Citizen Training Plan creation is essential. This work aims to show how vital participation is within processes for school educational improvement. The work methodology centers on reviewing specialized secondary sources, emphasizing the importance of involvement as a useful management tool. Evidence leads us to conclude that promoting active participation is an excellent challenge for educational leaders, which often means rallying principles associated with mutual collaboration among those involved in school government. This challenge lies in not proposing education participation based on "imposed" regulatory frameworks, be it a law or a decree. On the contrary, education communities should seek to promote collaborative and participatory actions based on self-management, taking into account their contexts and criteria, fostering the creation of high expectations, trust, and processes for improvement. school improvement, national policy of school coexistence, citizen training. 


\section{Introducción. La participación como herramienta de mejora}

La participación incluye múltiples formas (instancias formales e informales) en que se puede ser un aporte, entendiéndose como un proceso de involucramiento de los distintos actores de la comunidad escolar en las decisiones y acciones que los afectan a ellos o a su entorno. Esta puede darse de manera individual u organizada (Flamey, 2005, p. 3). La Ley General de Educación define la participación como un espacio en el que "los miembros de la comunidad educativa tienen derechos a ser informados y participar en el proceso educativo en conformidad a la normativa vigente" (LGE, 2009, p. 4). Sabemos, por la literatura internacional, que las visiones y expectativas acerca de la escuela se fortalecen cuando los líderes educativos promueven dinámicas en sus comunidades, para desarrollar relaciones de apoyo mutuo y propósitos compartidos (Leithwood \& Riehl, 2003, citado en Mineduc, 2016).

El presente estudio tiene como finalidad conocer e identificar algunas características de la participación educativa y su importancia para la mejora escolar. La metodología de trabajo consistió en la revisión de diversas fuentes secundarias especializadas en participación escolar (Ascorra, López \& Urbina, 2016; Aravena, Escare \& Ramírez, 2019; Fierro, 2013; Anderson, 2012; De Alba, García y Santisteban, 2012; Hart, 1993; Cerda, Egaña, Magendzo, Santa Cruz \& Varas, 2004; Leithwood \& Riehl, 2003; Muñoz, 2011; Tonon, 2012; Trilla \& Novella, 2001; entre otros), como también aquellas normativas establecidas por la regulación vigente en educación (Ministerio de Educación, 2016, 2017, 2019, 2015, s.f.; Ley 20.911, 2016; Ley 20.370, 2009, entre las más destacadas).

En función de lo anterior, se analiza en primer lugar la participación y su importancia para la mejora; en segundo, la participación y el rol de la gestión directiva en generar esos procesos; tercero, la participación y la política educativa, tomando en consideración la Política Nacional de Convivencia Escolar y el Plan de Formación Ciudadana, con fin de visualizar el impacto y la importancia de generar acciones que promuevan la participación educativa; finalmente, se reflexiona a partir del desafío que significa instalar acciones participativas.

La participación, asociada al mundo escolar, se ha venido trabajando fuertemente en las diversas investigaciones educativas durante el 
último tiempo. Actualmente, corresponde a un proceso fundamental para generar procesos de mejora educativa efectiva, quedando esto plasmado en una serie marcos normativos y legales de la política educativa. De acuerdo con un documento del Ministerio de Educación, "participar, en un establecimiento educacional, es integrarse activamente en la comunidad escolar, con el fin de aportar a un objetivo compartido por todos: el mejoramiento de la calidad de la educación" (Mineduc, 2016, p. 3).

La participación escolar está fuertemente asociada al concepto de "participación ciudadana" (Ascorra, López y Urbina, 2016) y/o "participación democrática" (Tonon, 2012), en tanto se espera que en la escuela los estudiantes puedan realizar el ejercicio de la ciudadanía, a través de su involucramiento activo en los procesos escolares y también de la convivencia escolar (Martínez, Silva, Morandé \& Canales, 2010; Schulz \& Fraillon, 2012; De Alba, García \& Santisteban, 2012). Por esto, la participación puede ser una práctica que no se presenta de la misma manera en todos los establecimientos, porque algunos atribuyen la participación a la promoción de la convivencia escolar y otros a la creación de instrumentos de gestión, como el Proyecto Educativo Institucional (PEI) (Mineduc, 2015).

\section{El liderazgo distribuido para la gestión efectiva y participación}

El trabajo en la escuela no puede ser considerado como un proceso individual, ya que se encuentra en la obligación permanente de establecer interacciones entre los diversos actores de la comunidad escolar, para cumplir con las distintas demandas que el cotidiano impone. Desde una perspectiva del liderazgo distribuido, trasciende de la figura del director y apunta a los distintos actores que, de una u otra forma, ejercen funciones de influencia en la organización escolar. Estos múltiples actores, capaces de influir y desplegar prácticas de liderazgo, van desde jefes técnicos, coordinadores de área o docentes (Anderson, 2012; Crawford, 2012; Spillane, 2006; Spillane, Diamond \& Jita, 2003).

El ejercicio del liderazgo efectivo en las escuelas tiene una relación directa con la participación, cumpliendo un rol de refuerzo de los principios democráticos sobre la toma de decisiones en la organización escolar (Fusarelli, Kowalski \& Petersen, 2011). Por consiguiente, 
un establecimiento que sabe escuchar a sus docentes, asistentes de la educación, estudiantes y familias, y que genera diversas instancias de participación, no se crea espontáneamente o por mero decreto, se conforma en la dinámica de las relaciones humanas que se establecen. (Mineduc, 2015, p. 18)

Es importante comprender que un proceso participativo necesita del diálogo y de generar espacios de reflexión crítica, que permitan deconstruir significados arraigados en la cultura de las organizaciones y en los propios sujetos (Muñoz, 2011), por eso la importancia de la gestión directiva.

En función de lo anterior, Trilla y Novella (2001) proponen distintos tipos de participación, entre los que se destacan: participación simple, participación consultiva, participación proyectiva y metaparticipación (tabla 1). Los autores establecen que, a medida que se avanza en estos niveles, va aumentando la complejidad de la participación.

Tabla 1.

Tipos de Participación

\begin{tabular}{ll}
\hline $\begin{array}{l}\text { Meta } \\
\text { Participación }\end{array}$ & $\begin{array}{l}\text { En este tipo de participación son las personas quienes } \\
\text { solicitan un cambio, es decir, consideran que sus derechos } \\
\text { participativos no son escuchados. }\end{array}$ \\
\hline $\begin{array}{l}\text { Participación } \\
\text { Proyectiva }\end{array}$ & $\begin{array}{l}\text { Se caracteriza por el desarrollo de una participación } \\
\text { activa, en la que el sujeto escolar es agente de las acciones } \\
\text { y se involucra de manera permanente en el desarrollo } \\
\text { de las mismas. En la participación proyectiva las ideas } \\
\text { planteadas por el adulto sufren modificaciones, siendo la } \\
\text { distorsión de la idea original una manifestación de este }\end{array}$ \\
& tipo de participación. \\
\hline Participación & $\begin{array}{l}\text { Estimula la participación. Se escucha la opinión de los } \\
\text { sujetos, pudiendo ser esta última vinculante o no. Es decir, } \\
\text { Consultiva }\end{array}$ \\
\hline Participación & $\begin{array}{l}\text { Se caracteriza por una participación que es medida en } \\
\text { cantidad. Es decir, simplemente "se está presente". }\end{array}$ \\
\hline
\end{tabular}

Fuente: Aravena, Escare y Ramírez (2019); Mineduc (2017).

Queda claro, a partir de la tabla 1, que no es solo la visión del director lo que influye en la promoción de cambios profundos dentro de la escuela; la participación cobra valor como una práctica que facilita la instalación del liderazgo distribuido. Sin duda, el rol de establecer 
visiones compartidas en las comunidades académicas es fundamental para la administración y el liderazgo educativo, y el hecho de que existan acciones o instancias participativas no asegura la participación de todos los actores de la comunidad escolar. Se enfatiza que

los espacios donde la participación de distintos actores es valorada y considerada en la toma de decisiones favorecen la generación de un clima escolar positivo y los sentimientos de pertenencia de las personas a una comunidad. De manera organizada, el director y su equipo directivo pueden promover la participación de los actores de la comunidad educativa mediante el trabajo efectivo del Consejo Escolar, el Consejo de Profesores, el Centro de Padres y Apoderados y el Centro de Estudiantes, entre otros. (Mineduc, 2016, p. 3).

Por esto, resulta trascendental generar e instaurar visiones compartidas para generar un aprendizaje organizacional y mayor participación de todos los actores de la comunidad educativa. Es imprescindible involucrar a los distintos actores de la comunidad escolar: estudiantes, familia, docentes y asistentes de la educación, etc., y, asimismo, facilitar el trabajo en conjunto para alcanzar los objetivos, fortaleciendo la visión del establecimiento educacional y articulando las decisiones impulsadas por los directivos (Mineduc, 2016; Castañeda \& Fernández, 2007; Bellei et al., 2014). No obstante, se debe prever que estos espacios de participación en la toma de decisiones no deben estar sujeto a un modo de participación clientelista (Muñoz, 2011).

\section{La participación en la política educativa}

Como fue mencionado, "participar consiste en el involucramiento y colaboración de las y los integrantes de la comunidad educativa en un marco de respeto, permitiendo que la gestión global de la misma cuente con mayores niveles de legitimidad" (Mineduc, 2017, p. 15). En el caso de las políticas educativas, estas han estado en permanente evolución, vinculadas a los cambios de nuestro entorno social, cultural, político, económico e institucional. Así, mientras en el siglo pasado la toma de decisiones era exclusivamente estatal, con poder de crear, estructurar y modificar el ciclo de las políticas públicas, en la actualidad la participación de diversos actores, la creación de consenso y la legitimidad en los asuntos públicos se han tornado 
ineludibles para la gobernanza en cualquier territorio (Lahera, 2008; Espinoza, 2009; Aziz, 2018). En ese sentido, las políticas públicas son instrumentos de gobernabilidad que dan forma a la política, asignando ganadores y perdedores entre ciudadanos y grupos de interés, lo que suele afectar el ciclo de política, la participación y las demandas futuras (May \& Jochim, 2013). En el caso de Chile, la participación de distintos actores en los marcos legales de la educación tiene su inicio en 2006, con mayor preponderancia de marchas estudiantiles y el involucramiento de la sociedad en general. A partir de esa fecha inició un periodo de participación de actores claves en la elaboración de las políticas, lo que influenció varias leyes fundamentales que dieron base a una reforma estructural del sistema educativo chileno, como la Subvención Escolar Preferencial (SEP), la Ley General de Educación (LGE), la Ley de Aseguramiento de la Calidad (SAC), la Ley de Inclusión, la Ley de Carrera Docente y la Ley que crea el nuevo Sistema de Educación Pública, la Política Nacional de Convivencia Escolar (PNCE) y el desarrollo de la Formación Ciudadana (PFC), entre otras (Aziz, 2018).

A raíz de lo anterior, se tomará la actual Política Nacional de Convivencia Escolar (PNCE) y el Plan de Formación Ciudadana (PFC) como como ejemplo de procesos de participación activa por parte de las comunidades educativas y sus implicancias en la mejora.

\section{Política Nacional de Convivencia Escolar (PNCE)}

La reciente Política Nacional de Convivencia Escolar la define como "el conjunto de las interacciones y relaciones que se producen entre todos los actores de la comunidad" (PNCE, 2019, p. 9, citado en Aravena et al., 2019). "Dentro de este marco, se establece que la convivencia es un proceso permanente de aprendizaje y enseñanza, y una construcción sociocultural, de carácter dinámico y cotidiano" (Aravena et al., 2019, p. 2). Al respecto,

se espera de esta política sea una herramienta que aporte a la gestión de la convivencia y a "una toma de conciencia sobre la materia de todos los miembros de la comunidad educativa: equipos directivos, docentes, asistentes de la educación, padres y apoderados y, por supuesto, los más de 3,5 millones de estudiantes que existen en Chile. (PNCE, 2019, p. 7) 
La PNCE tiene cuatro características que permiten la promoción de la convivencia escolar en los establecimientos educacionales.

Una convivencia Inclusiva

Caracterizada por la participación democrática.
Una convivencia basada en un trato respetuoso.
Resolución pacífica de los conflictos

Figura 1. Ejes de la Política Nacional de Convivencia Escolar (2019) Fuente: Elaboración propia, a partir PNCE (2019).

Se espera que esta actualización de la política de convivencia escolar modifique los procesos de toma de decisión a partir de la participación de todos sus actores. Es de vital importancia la labor que desempeña el encargado de Convivencia Escolar en los establecimientos educacionales, quien debe ejercer una función de "espónsor", liderando y gestionando el cambio a partir de los ejes establecidos en la política. Debe ofrececerse oportunidades para la expresión de opiniones, la deliberación y el debate fundamentado de ideas, la construcción de acuerdos y el respeto de estos por parte de toda la comunidad, y la colaboración necesaria para llevar adelante las distintas actividades del quehacer cotidiano (PNCE, 2019).

Desde el punto de vista de la participación, "se busca promover la colaboración que facilite la construcción de un sentido de pertenencia, basado en una identificación positiva con la comunidad educativa, su cultura y las actividades que en ella se realizan" (PNCE, 2019, p. 14).

Desde una mirada organizacional, la gestión debe ser coherente y pertinente, pues busca unir las declaraciones conceptuales, el tipo de convivencia que se quiere promover y las características del contexto específico de cada establecimiento y sus actores, con los objetivos y ac- 
ciones que se diseñen e implementen (PNCE, 2019, p. 16; Fierro, 2013; Mineduc, 2015).

En consecuencia, el desafío es propiciar, dinamizar y permitir el funcionamiento de estas instancias, de manera de constituir comunidades que se gestionan y se recrean en un proceso participativo de autoconstrucción.

\section{Plan de Formación Ciudadana (PFC)}

La participación en el aula es vital para el desarrollo ciertos aprendizajes en ciudadanía. Estas prácticas escolares deben ser entes colaboradores en la enseñanza y el aprendizaje, y en la interacción (Mineduc, 2017). Al respecto, fortalecer preguntas reflexivas dentro del aula, lluvia de ideas, definir con propias palabras, participar vía votos, expresar sentimientos y afectos, solicitar opiniones personales, elaborar pensamientos complejos, que los estudiantes propongan formas de trabajo, son algunas de las estrategias que tienen buenos resultados.

Gracias a la Ley 20.911 se posiciona en el currículo la inclusión de la formación ciudadana. Llama la atención en la Ley la amplitud de sus propósitos: "promover" y "fomentar" son los más utilizados para dar sentido a un conjunto de significados ya presentes en nuestros currículos: ciudadanía, derechos y deberes, Estado de Derecho, instituciones, virtudes cívicas, derechos humanos, Constitución política, tratados internacionales, derechos del niño, diversidad, interés en lo público y cultura democrática (Henríquez y Rasse, 2018).

En este contexto, la Ley 20.911, que crea el Plan de Formación Ciudadana, invita a las escuelas y liceos a intencionar este diálogo. Para ello, establece que:

Los establecimientos educacionales reconocidos por el Estado deberán incluir en los niveles de enseñanza parvularia, básica y media un Plan de Formación Ciudadana, que integre y complemente las definiciones curriculares nacionales en esta materia, que brinde a los estudiantes la preparación necesaria para asumir una vida responsable en una sociedad libre y dé orientación hacia el mejoramiento integral de la persona humana, como fundamento del sistema democrático, la justicia social y el progreso. Asimismo, deberá propender a la formación de ciudadanos, con valores y co- 
nocimientos para fomentar el desarrollo del país, con una visión del mundo centrada en el ser humano, como parte de un entorno natural y social. En el caso de la educación parvularia, este plan se hará de acuerdo a las características particulares de este nivel y su contexto, por ejemplo, a través del juego. (Mineduc, s.f., p. 8).

Con el fin de alcanzar lo anterior, se proponen los siguientes objetivos a desarrollar en las escuelas y liceos:

a) Promover la comprensión y análisis del concepto de ciudadanía y los derechos y deberes asociados a ella, entendidos éstos en el marco de una república democrática, con el propósito de formar una ciudadanía activa en el ejercicio y cumplimiento de estos derechos y deberes. b) Fomentar en los estudiantes el ejercicio de una ciudadanía crítica, responsable, respetuosa, abierta y creativa. c) Promover el conocimiento, comprensión y análisis del Estado de Derecho y de la institucionalidad local, regional y nacional, y la formación de virtudes cívicas en los estudiantes. d) Promover el conocimiento, comprensión y compromiso de los estudiantes con los derechos humanos reconocidos en la Constitución Política de la República y en los tratados internacionales suscritos y ratificados por Chile, con especial énfasis en los derechos del niño. e) Fomentar en los estudiantes la valoración de la diversidad social y cultural del país. f) Fomentar la participación de los estudiantes en temas de interés público. g) Garantizar el desarrollo de una cultura democrática y ética en la escuela. h) Fomentar una cultura de la transparencia y la probidad. i) Fomentar en los estudiantes la tolerancia y el pluralismo. (Ley 20.911, 2016).

Uno de los aspectos relevantes es la participación de la comunidad educativa. De acuerdo con Cerda et al. (2004), es necesario que se establezcan dinámicas de discusión sobre formación ciudadana en el establecimiento educacional asociadas a: a) el imperativo del cambio, b) necesidad de introducir una mirada ética en la formación ciudadana, c) importancia de lo público, d) expandir la mirada crítica, e) el currículo y las prácticas, f) convertir las prácticas cotidianas en motivo de reflexión y observación para estudiantes y profesores.

Por un lado, no se debe caer en malas interpretaciones, teniendo en cuenta que realizar un Plan de Formación Ciudadana en la escue- 
la no garantiza que se esté promoviendo realmente el desarrollo de la Formación Ciudadana de una manera íntegra. No consiste en crear un instrumento que sirva solo para rendir cuentas (acountability), sino que permita efectivamente la formación ciudadanos activos, críticos, sociales y políticos (Loyola, 2019).

Por otro lado, es necesario que los equipos directivos, en su gestión y liderazgo, no establezcan limitaciones institucionales asociadas a políticas de rendición de cuentas, ya que impondrían a los docentes mayor carga de tiempo y escasos momentos de reflexión y participación escolar. De ahí la importancia de tomar decisiones acordes a los objetivos planeados en el Proyecto Educativo Institucional.

Respecto de los espacios de participación (figura 2) se debe tener claridad acerca de aquellos espacios (formales) de participación en las escuelas, entre los que destacamos el aula y los con la comunidad. Es fundamental, y a la vez una tarea, que los establecimientos fortalezcan espacios reales de encuentro en que se respeten los aportes, experiencias y atribuciones de cada actor.

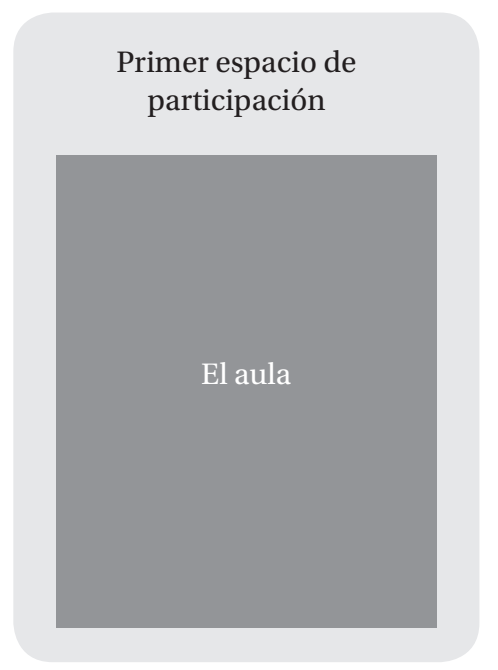

\section{Espacios de participación en las comunidades educativas}

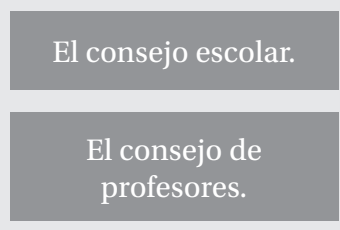

El centro de alumnos

La reunión de

apoderados(as

Figura 2. Espacios de participación existentes en la unidad educativa Fuente: elaboración propia (2020).

Tal como se visualiza, se espera que se establezcan no solo espacios formales (figura 2) de participación como los nombrados, sino que esto incluya también espacios informales, como actividades recreacio- 
nales, y extracurriculares, iniciativas de apertura a la comunidad y una planificación curricular que refuerce el desarrollo de la ciudadanía, la ética y la cultura democrática en los diversos talleres de carácter formativo.

A partir de lo anterior, ocupando estos espacios de participación en la unidad educativa, a continuación se presentan siete recomendaciones para desarrollar prácticas efectivas de participación promovidas por los líderes educativos.

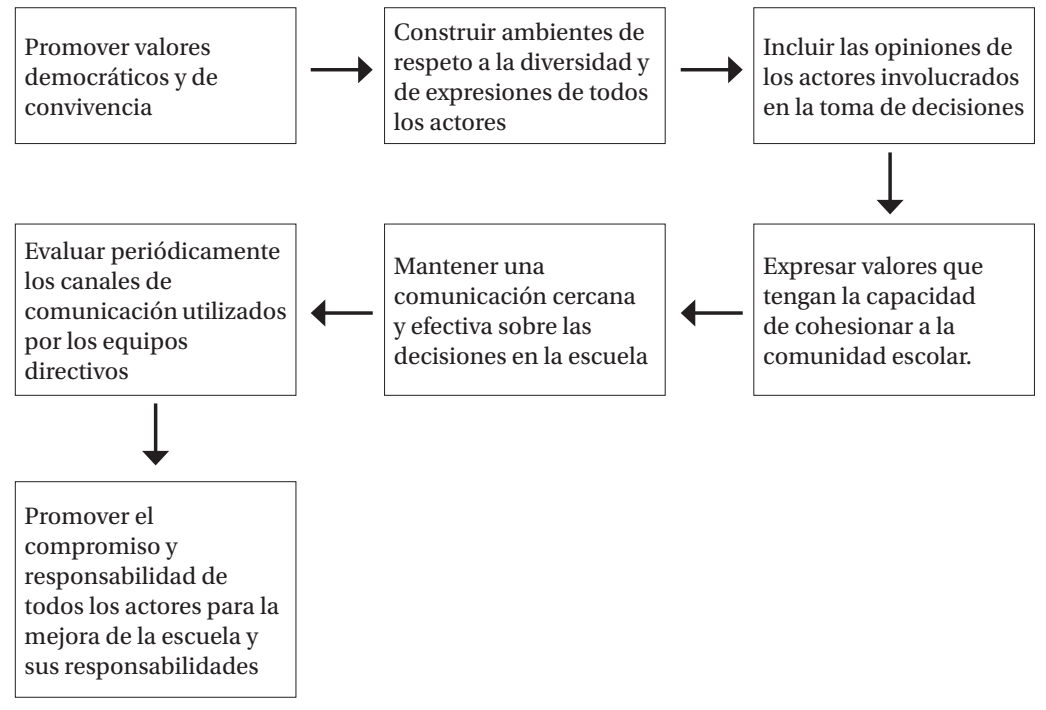

Figura 3. Prácticas que fomentan la participación en la escuela Fuente: elaboración propia (2020).

Al respecto, el Proyecto Educativo Institucional representa, desde sus propósitos, una instancia de participación y representatividad, visión compartida, promoción de prácticas democráticas y fortalecimiento de una sana convivencia entre todos los integrantes. Por esta razón se vuelve relevante analizar la función que este instrumento cumple en el proceso de mejora.

La cooperación y colaboración son elementos que van de la mano con la participación; ello permite la cohesión y sinergia en la comunidad escolar. Sin duda, el monitoreo es esencial, desde la perspectiva de la gestión, para evaluar que las acciones y actividades están teniendo un impacto positivo en la organización escolar. Es importante recalcar 
que el compromiso y establecer metas y expectativas son esenciales para fomentar procesos de mejora escolar. Finalmente, con estas actividades se logra fomentar la participación de todos los estudiantes, desarrollando habilidades comunicacionales y sociales, e incorporando la presencia activa de apoderados en el proceso de aprendizaje de los estudiantes.

\section{Conclusiones}

Se aprecia un esfuerzo, por parte del Ministerio de Educación, en la Política Nacional de Convivencia Escolar y en la Formación Ciudadana, entre otros instrumentos, en los que se pretende fomentar la participación en la comunidad escolar tomando en consideración a todos los actores. Sin embargo, lograr una participación activa genera un desafío potente para los líderes educativos, porque implica movilizar creencias asociadas a la colaboración mutua entre los que están insertos en la organización escolar.

El desafío de la participación educativa está en no plantearla a partir de marcos normativos "impuestos", sea esta una ley o decreto, sino que, por el contrario, apostar a que las comunidades educativas busquen la promoción de acciones colaborativas y participativas a partir de la autogestión, tomando sus contextos y criterios, favoreciendo a la creación de altas expectativas, confianza y procesos de mejora. Entendemos que la participación educativa corresponde a una herramienta muy significativa para la mejora escolar. Así lo demuestra la evidencia especializada. De ahí surge la necesidad constante de crear, innovar y establecer espacios de participación en la comunidad educativa, tal como se hace en la experiencia educativa. Los resultados y logros se relacionan con la creación de espacios de confianza entre el equipo directivo, los docentes, los estudiantes y los apoderados, porque la participación de las familias potencia las iniciativas y la articulación de las asignaturas.

A partir de lo anterior, la participación es también en un proceso formativo de enseñanza-aprendizaje, mediante el cual se puede facilitar la construcción de valores éticos y democráticos para la formación ciudadana. La consolidación de principios éticos nos conduce a pensar en una ética democrática en la que el ciudadano participa en la transformación de su medio (Muñoz, 2013). Al respecto, Freire 
(2002) afirma que nos sentimos parte de un conflicto cuando la participación se da en condiciones de igualdad, cuando profundizamos en el diálogo y cuando analizamos y revisamos la realidad para posteriormente transformarla. En este sentido, el diálogo supone fortalecer las condiciones de igualdad entre las personas que lo integran, pero se necesita asegurar unas condiciones para construir consenso en dicho diálogo.

Finalmente, es necesario establecer diversos canales de participación en la escuela, en los que se funciones instancias efectivas, tanto de comunicación como de colaboración. Si bien cada establecimiento educacional posee asociaciones formales de participación — sin duda eficientes-, también se debe visualizar instancias informales, con el objetivo de buscar distintas metodologías para recoger apreciaciones de todos los actores de la comunidad educativa. Para esto se sugiere considerar los tipos y niveles de participación que se dan en un establecimiento educacional, como también las prácticas y acciones para fundar procesos de mejora escolar.

\section{Referencias bibliográficas}

Anderson, M. (2012). The Struggle for Collective Leadership: Thinking and Practice in a Multicampus School Setting. Educational Management Administration \& Leadership, 40(3), 328-342.

Aravena, F., Escare, K. y Ramírez, J. (2019). La participación democrática y la voz de los estudiantes: ¿Qué pueden hacer los líderes escolares? Nota Técnica $N^{o}$ 2. Líderes educativos. Valparaíso: Centro de Liderazgo para la Mejora Escolar:

Ascorra, P., López, V. y Urbina, C. (2016). Participación estudiantil en escuelas chilenas con buena y mala convivencia escolar. Revista de Psicología, 25(2), 1-18.

Aziz, C. (2018). Evolución e implementación de las políticas educativas en Chile. Nota técnica № 2. Líderes educativos. Valparaíso: Centro de Liderazgo para la Mejora Escolar.

Bellei, C., Valenzuela, J., Vanni, X. \& Contreras, D. (2014). Lo aprendí en la escuela: ¿Cómo se logran procesos de mejoramiento escolar? Santiago de Chile: LOM.

Biblioteca del Congreso Nacional de Chile. (2016). Ley 20.911 (2016). Crea el plan de Formación Ciudadana para los estableci- 
mientos educacionales reconocidos por el Estado. Recuperado de https://www.leychile.cl/Navegar?idNorma=1088963

Biblioteca del Congreso Nacional de Chile. (2009). Ley General de Educación $\mathrm{N}^{\circ}$ 20370. Recuperado de https://www.leychile.cl/Na vegar?idNorma $=1006043 \& i d P a r t e=$

Castañeda, D. I. \& Fernández, M. (2007). Validación de una escala de niveles y condiciones de aprendizaje organizacional. Universitas Psychológica, 6(2), 245-254.

Cerda, A., Egaña, M., Magendzo, A., Santa Cruz, E. y Varas, R., (2004). El Complejo Camino de la Formación ciudadana, Santiago de Chile: LOM.

Crawford, M. (2012). Solo and Distributed Leadership: Definitions and Dilemmas. Educational Management Administration \& Leadership, 40(5), 610-620.

De Alba, N., García, F. y Santisteban, A. (2012). Educar para la participación ciudadana en la enseñanza de las ciencias sociales. Sevilla: Asociación Universitaria de Profesorado de Didáctica de las Ciencias Sociales.

Espinoza, O. (2009). Reflexiones sobre los conceptos de "política", políticas públicas y política educacional. Archivos Analíticos de Políticas Educativas, 17(8).

Fierro, M. C. (2013) Convivencia inclusiva y democrática. Una perspectiva para gestionar la seguridad escolar. Revista Electrónica Sinéctica, 40, 1-18.

Flamey, G. et al. (2005). Participación de los Centros de Padres en la Educación: Ideas y Herramientas para mejorar la organización. Santiago de Chile: UNICEF.

Freire, P. (2002). Pedagogía de la esperanza. Un reencuentro con la pedagogía del oprimido. Argentina: Siglo XXI.

Fusarelli, I., Kowalski, T. \& Petersen, G. (2011). Distributive leadership, civic engagement, and deliberative democracy as vehicles for School Improvement. Leadership and Policy in Schools, 10(1), 43-62.

Hart, R. A. (1993). Children's participation: From tokenism to citizenship. Florence: Unicef, International Child Development Center.

Henríquez, R. y Rasse, C. (2018). Desafíos de la Formación Ciudadana para su Enseñanza y Aprendizaje, en I. Sánchez (ed.), Ideas 
en Educación II. Definiciones en tiempos de cambio. Santiago de Chile: CEPPE, Ediciones UC.

Lahera, E. (2008). Introducción a las políticas públicas. Santiago de Chile: Fondo de Cultura Económica.

Leithwood, K. A. \& Riehl, C. (2003). What do we already know about successful school leadership? Washington, DC: AERA Division A task force on developing research in educational leadership.

Loyola, C. (2019). Gestionando desde dentro la Formación Ciudadana. Tesis de postgrado. Viña del Mar: Pontificia Universidad Católica de Valparaíso.

Martínez, M. L., Silva, C., Morandé, M. \& Canales, L. (2010). Los jóvenes ciudadanos: reflexiones para una política de formación ciudadana juvenil. Última Década, 32, 105-118.

May, P. J. y Jochim, A. E. (2013). Policy regime perspectives: Policies, politics and governing. Policy Studies Journal, 41(3).

Ministerio de Educación. (2015). Marco para la Buena Dirección y el Liderazgo Escolar. Santiago de Chile.

Ministerio de Educación. (2016). Gestión de la participación de la comunidad escolar. Dimensión: Gestionando la convivencia y la participación de la comunidad escolar. Santiago de Chile.

Ministerio de Educación. (2017). Orientaciones para la participación de las Comunidades Educativas en el Marco del Plan de Formación Ciudadana. Santiago de Chile.

Ministerio de Educación. (2019). Política Nacional de Convivencia Escolar (PNCE). Recuperado desde: http://convivenciaescolar. mineduc.cl/politica-nacional/

Ministerio de Educación. (s.f.) ¿Por qué un plan de formación ciudadana en la escuela? Conversemos: Cuaderno Docente $N^{o} 7$, División de Educación General.

Muñoz, G. (2011). La democracia y la participación en la escuela: ¿Cuánto se ha avanzado desde la normativa para promover la participación escolar? Revista de Estudios y Experiencias en Educación, 10(19), 107-129.

Schulz, W. \& Fraillon, J. (April, 2012). Students' participation in and valuing of civic engagement at school. Annual Meetings of the American Educational Research Association, Vancouver, Canada. 
Spillane, J. (2006). Distributed leadership. San Francisco: Jossey-Bass.

Spillane, J., Diamond, J. \& Jita, L. (2003). Leading instruction: the distribution of leadership for instruction. Journal of Curriculum Studies, 35(5), 533-543.

Tonon, G. (2012). Young people's quality of life and construction of citizenship. Netherlands: Springer.

Trilla, J. y Novella, A. (2001). Educación y participación social de la infancia. Revista Iberoamericana de Educación, 26, 1-21. 